\title{
ON QUATERNION ALGEBRAS OVER THE COMPOSITE OF QUADRATIC NUMBER FIELDS
}

\author{
Vincenzo Acciaro, Diana Savin, Mohammed \\ TaOus and ABDelKader ZeKhnini \\ Università di Chieti-Pescara, Italy, Transilvania University of Brașov, \\ Romania, Moulay Ismail University, Morocco and Mohammed First \\ University, Morocco
}

AbStract. Let $p$ and $q$ be two positive prime integers. In this paper we obtain a complete characterization of division quaternion algebras $H_{K}(p, q)$ over the composite $K$ of $n$ quadratic number fields.

\section{Introduction}

Let $F$ be a field with $\operatorname{char}(F) \neq 2$ and let $a, b \in F \backslash\{0\}$. The generalized quaternion algebra $H_{F}(a, b)$ over the field $F$ is the algebra having basis $\{1, i, j, k\}$ and multiplication table:

\begin{tabular}{c||c|c|c|c|}
$\cdot$ & 1 & $i$ & $j$ & $k$ \\
\hline \hline 1 & 1 & $i$ & $j$ & $k$ \\
\hline$i$ & $i$ & $a$ & $k$ & $a j$ \\
\hline$j$ & $j$ & $-k$ & $b$ & $-b i$ \\
\hline$k$ & $k$ & $-a j$ & $b i$ & $-a b$ \\
\hline
\end{tabular}

If $x=x_{1} 1+x_{2} i+x_{3} j+x_{4} k \in H_{F}(a, b)$, with $x_{i} \in F$, the conjugate $\bar{x}$ of $x$ is defined as $\bar{x}=x_{1} 1-x_{2} i-x_{3} j-x_{4} k$, and the norm of $x$ as $\boldsymbol{n}(x)=x \bar{x}=$ $x_{1}^{2}-a x_{2}^{2}-b x_{3}^{2}+a b x_{4}^{2}$.

2020 Mathematics Subject Classification. 11R04, 11R11, 11R21, 11R32, 11R52, 11S15, 11R37, 11R29, 11A41, 11F85.

Key words and phrases. Quaternion algebras, quadratic fields, biquadratic fields, composite of quadratic fields, ramification theory in algebraic number fields. 
Quaternion algebras turn out to be central simple algebras over $F$ (i.e. associative and noncommutative algebras without two sided ideals whose center is precisely $F$ ) of dimension 4 over $F$. Recall that the dimension $d$ of a central simple algebra $A$ over a field $F$ is always a perfect square, and its square root $n$ is defined to be the degree of $A$.

The theory of central simple algebras (in particular quaternion algebras and cyclic algebras) has strong connections with algebraic number theory, combinatorics, algebraic geometry, coding theory, computer science and signal theory.

If the equations $a x=b, y a=b$ have unique solutions for all $a, b \in A, a \neq$ 0 , then the algebra $A$ is called a division algebra. If $A$ is a finite-dimensional algebra, then $A$ is a division algebra if and only if $A$ has no zero divisors $(x \neq 0, y \neq 0 \Rightarrow x y \neq 0)$. In the case of generalized quaternion algebras there is a simple criterion that guarantees them to be division algebras: $H_{F}(a, b)$ is a division algebra if and only if there is a unique element of zero norm, namely $x=0$.

Let $L$ be an extension field of $F$, and let $A$ be a central simple algebra over $F$. We recall that $A$ is said to split over $L$, and then $L$ is called a splitting field for $A$, if $A \otimes_{F} L$ is isomorphic to a matrix algebra over $L$. For example, every quaternion algebra over $\mathbb{C}$ (or over any algebraically closed field) splits, and a quaternion algebra $H_{\mathbb{R}}(\alpha, \beta)$ over $\mathbb{R}$ splits if and only if $\alpha>0$ or $\beta>0$.

Several results are known about the splitting behavior of quaternion algebras over specific fields $([10,15,5,6,20])$. Explicit conditions which guarantee that a quaternion algebra splits over the field of rationals numbers, or else is a division algebra, were studied in [3]. In [22] the second author studied the splitting behavior of some quaternion algebras over some specific quadratic and cyclotomic fields. Moreover, in [23] the second author found some sufficient conditions for a quaternion algebra to split over a quadratic field.

Traditionally, in order to decide whether a quaternion algebra is division algebra or else it splits, either we look for the primes which ramify in the algebra or otherwise we appeal to Hasse's norm theorem which allows us to reduce the problem to the computation of local Hilbert symbols $([24,25])$. Very recently Goldstein and Schacher ([11]) tried to combine these two techniques.

Throughout this paper, all the fields are tacitly assumed to be of characteristic not 2 .

In this paper we adopt the former approach, i.e., we study the ramification of certain integral primes, and we obtain a nice characterization of quaternion division algebras $H_{K}(p, q)$ solely in terms of quadratic residues, assuming that $p$ and $q$ are positive primes and $K$ is a composite of $n$ quadratic number fields.

The structure of this paper as follows. In Section 2 we state some results about quaternion algebras and quadratic fields which we will need later. In Section 3 we collect some results about quaternion algebras over quadratic 
fields which were proved in a previous paper of ours ([1]). In Section 4 we find some necessary and sufficient conditions for a quaternion algebra over a biquadratic field to be a division algebra. In Section 5 we extend the previous results to any composite of $n$ quadratic number fields. In the last section we compare our approach with the classical ones, from a computational point of view.

The main result of this paper is the next theorem, which will be proved at the end of Section 5. In this theorem, $(\dot{\bar{p}})$ is the Legendre symbol. We recall the definition of the Legendre symbol in the preliminaries section.

MAIN THEOREM (Classification over the composite of $n$ quadratic fields). Let $d_{1}, \ldots, d_{n}$ be distinct squarefree integers not equal to one, multiplicatively independent over $\mathbb{Q}$, with $n \geq 2$. Let $p$ and $q$ be distinct odd positive prime integers such that $p$ does not divide $d_{i}$ and $q$ does not divide $d_{i}(i=1, \ldots, n)$. Let $K=\mathbb{Q}\left(\sqrt{d_{1}}, \ldots, \sqrt{d_{n}}\right)$ and $K_{i}=\mathbb{Q}\left(\sqrt{d_{i}}\right)(i=1, \ldots, n)$, with discriminant $\Delta_{K_{i}}$. Then the quaternion algebra $H_{K}(p, q)$ is a division algebra if and only if one of the following conditions holds:

(i) $p$ and $q$ are odd and distinct, and $\left(\frac{p}{q}\right)=-1$, and

$p \equiv 1(\bmod 4)$ or $q \equiv 1(\bmod 4)$, and

$\left(\frac{\Delta_{K_{1}}}{p}\right)=\ldots=\left(\frac{\Delta_{K_{n}}}{p}\right)=1$ or $\left(\frac{\Delta_{K_{1}}}{q}\right)=\ldots=\left(\frac{\Delta_{K_{n}}}{q}\right)=1$;

(ii) $q=2$, and

$p \equiv 3(\bmod 8)$ or $p \equiv 5(\bmod 8)$, and

either $\left(\frac{\Delta_{K_{1}}}{p}\right)=\ldots=\left(\frac{\Delta_{K_{n}}}{p}\right)=1$ or $d_{1}, \ldots, d_{n} \equiv 1(\bmod 8)$;

(iii) $p$ and $q$ are odd, with $p \equiv q \equiv 3(\bmod 4)$, and

- $\left(\frac{q}{p}\right) \neq 1$, and

- either $\left(\frac{\Delta_{K_{1}}}{p}\right)=\ldots=\left(\frac{\Delta_{K_{n}}}{p}\right)=1$ or $d_{1}, \ldots, d_{n} \equiv 1(\bmod 8)$;

or

- $\left(\frac{p}{q}\right) \neq 1$, and

- either $\left(\frac{\Delta_{K_{1}}}{q}\right)=\ldots=\left(\frac{\Delta_{K_{n}}}{q}\right)=1$ or $d_{1}, \ldots, d_{n} \equiv 1(\bmod 8)$.

\section{Preliminaries}

In this section we recall some basic facts about quadratic and biquadratic fields, as well as some important results concerning quaternion algebras. Unless otherwise stated, when we say a prime integer we mean a positive prime integer. If $a$ is an integer and $p$ is an odd prime integer, we denote with $\left(\frac{a}{p}\right)$ the Legendre symbol of $a$ modulo $p$. We recall that

$\left(\frac{a}{p}\right)= \begin{cases}1, & \text { if } p \text { does not divide } a \text { and } a \text { is a quadratic residue mod } p ; \\ -1, & \text { if } p \text { does not divide } a \text { and } a \text { is a quadratic nonresidue mod } p \\ 0, & \text { if } p \mid a .\end{cases}$ 
Let us recall first the decomposition behavior of an integral prime ideal in the ring of integers of quadratic number fields ([12, Chapter 13]).

TheOREm 2.1 (Decomposition of primes in quadratic fields). Let $d \neq 0,1$ be a square free integer. Let $\mathcal{O}_{K}$ be the ring of integers of the quadratic field $K=\mathbb{Q}(\sqrt{d})$ and $\Delta_{K}$ be the discriminant of $K$. Let $p$ be an odd prime integer. Then, we have:

(i) $p$ is ramified in $\mathcal{O}_{K}$ if and only if $p \mid \Delta_{K}$. In this case $p \mathcal{O}_{K}=(p, \sqrt{d})^{2}$;

(ii) $p$ splits totally in $\mathcal{O}_{K}$ if and only if $\left(\frac{\Delta_{K}}{p}\right)=1$. In this case $p \mathcal{O}_{K}=$ $P_{1} \cdot P_{2}$, where $P_{1}$ and $P_{2}$ are distinct prime ideals in $\mathcal{O}_{K}$;

(iii) $p$ is inert in $\mathcal{O}_{K}$ if and only if $\left(\frac{\Delta_{K}}{p}\right)=-1$;

(iv) the prime 2 is ramified in $\mathcal{O}_{K}$ if and only if $d \equiv 2(\bmod 4)$ or $d \equiv 3$ $(\bmod 4)$. In the first case $2 \mathcal{O}_{K}=(2, \sqrt{d})^{2}$, while in the second case $2 \mathcal{O}_{K}=(2,1+\sqrt{d})^{2}$

(v) the prime 2 splits totally in $\mathcal{O}_{K}$ if and only if $d \equiv 1(\bmod 8)$. In this case $2 \mathcal{O}_{K}=P_{1} \cdot P_{2}$, where $P_{1}, P_{2}$ are distinct prime ideals in $\mathcal{O}_{K}$, with $P_{1}=\left(2, \frac{1+\sqrt{d}}{2}\right)$;

(vi) the prime 2 is inert in $\mathcal{O}_{K}$ if and only if $d \equiv 5(\bmod 8)$.

We recall that the discriminant $\Delta_{K}$ of a quadratic field $K=\mathbb{Q}(\sqrt{d})$ is

$$
\Delta_{K}= \begin{cases}4 d, & \text { if } d \equiv 2 \text { or } 3 \quad(\bmod 4) ; \\ d, & \text { if } d \equiv 1 \quad(\bmod 4)\end{cases}
$$

(see $[12, \mathrm{p} .189])$.

Now, let $d_{1}$ and $d_{2}$ be two distinct squarefree integers not equal to one. It is well known that $K=\mathbb{Q}\left(\sqrt{d_{1}}, \sqrt{d_{2}}\right)$ is a Galois extension of $\mathbb{Q}$ with Galois group isomorphic to the Klein 4-group. There are three quadratic subfields of $K$, namely $\mathbb{Q}\left(\sqrt{d_{1}}\right), \mathbb{Q}\left(\sqrt{d_{2}}\right)$ and $\mathbb{Q}\left(\sqrt{d_{3}}\right)$, where $d_{3}=\operatorname{lcm}\left(d_{1}, d_{2}\right) / \operatorname{gcd}\left(d_{1}, d_{2}\right)$. The next result concerning prime ideals which split completely in composita of extensions of the base field is quite general; we state it only in the case of our interest, i.e. for biquadratic number fields ([19, p. 46]), which are the composita of two quadratic number fields.

ThEOREM 2.2 (Splitting of primes in biquadratic fields). Let $d_{1}$ and $d_{2}$ be two distinct squarefree integers not equal to one, and let $d_{3}=$ $\operatorname{lcm}\left(d_{1}, d_{2}\right) / \operatorname{gcd}\left(d_{1}, d_{2}\right)$. Let $\mathcal{O}_{K}$ denote the ring of integers of the biquadratic field $K$ and $\mathcal{O}_{K_{i}}$ the ring of integers of the quadratic subfield $K_{i}=$ $\mathbb{Q}\left(\sqrt{d_{i}}\right), i=\{1,2,3\}$. Let $p$ be a prime integer. Then $p$ splits completely in $\mathcal{O}_{K}$ if and only if $p$ splits completely in each $\mathcal{O}_{K_{i}}(i=\{1,2,3\})$.

Next, let $K$ be a number field and let $\mathcal{O}_{K}$ be its ring of integers. If $v$ is a place of $K$, let us denote by $K_{v}$ the completion of $K$ at $v$. We recall that a quaternion algebra $H_{K}(a, b)$ is said to ramify at a place $v$ of $K$ - or $v$ is said to ramify in $H_{K}(a, b)$ - if the quaternion $K_{v}$-algebra $H_{v}=K_{v} \otimes H_{K}(a, b)$ is 
a division algebra. This happens exactly when the Hilbert symbol $(a, b)_{v}$ is equal to -1 , i.e. when the equation $a x^{2}+b y^{2}=1$ has no solutions in $K_{v}$. We recall that the reduced discriminant $D_{H_{K}(a, b)}$ of the quaternion algebra $H_{K}(a, b)$ is defined as the product of those prime ideals of the ring of integers $\mathcal{O}_{K}$ of $K$ which ramify in $H_{K}(a, b)$. The following splitting criterion for a quaternion algebras is well known ([3, Corollary 1.10]).

Proposition 2.3. Let $K$ be a number field and let $a, b \in K$ such that $a$ and $b$ are totally positive in $K$. Then, the quaternion algebra $H_{K}(a, b)$ is split if and only if its discriminant $D_{H_{K}(a, b)}$ is equal to the ring of integers $\mathcal{O}_{K}$ of $K$.

If $\mathcal{O}_{K}$ is a principal ideal domain, then we may identify the ideals of $\mathcal{O}_{K}$ with their generators, up to units. Thus, in a quaternion algebra $H$ over $\mathbb{Q}$, the element $D_{H}$ turns out to be an integer, and $H$ is split if and only if $D_{H}=1$.

The next proposition gives us a geometric interpretation of splitting ([10, Proposition 1.3.2]).

Proposition 2.4. Let $K$ be a field. Then, the quaternion algebra $H_{K}(a, b)$ is split if and only if the conic $a x^{2}+b y^{2}=z^{2}$ has a rational point in $K$, i.e. there are $x_{0}, y_{0}, z_{0} \in K$ such that $a x_{0}^{2}+b y_{0}^{2}=z_{0}^{2}$.

The next proposition due to Hasse relates the norm group of extensions of the base field to the splitting behavior of a quaternion algebra ([10, Proposition 1.1.7]).

Proposition 2.5. Let $F$ be a field. Then, the quaternion algebra $H_{F}(a, b)$ is split if and only if $a$ is the norm of an element of $F(\sqrt{b})$.

Fortunately, for the quaternion algebras it is true the following ([10, Proposition 1.1.7]).

Proposition 2.6. Let $K$ be a field and let $\alpha, \beta \in K \backslash\{0\}$. Then the quaternion algebra $H_{K}(\alpha, \beta)$ is either split or a division algebra.

In particular, this tells us that a quaternion algebra $H_{\mathbb{Q}}(a, b)$ is a division algebra if and only if there is a prime $p$ such that $p \mid D_{H_{\mathbb{Q}}(a, b)}$.

We end up this section with two statements following from the classical Albert-Brauer-Hasse-Noether theorem. Proofs of specific formulations of this theorem can be found in $[18,7]$.

THEOREM 2.7. Let $H_{F}$ be a quaternion algebra over a number field $F$ and let $K$ be a quadratic extension of $F$. Then there is an embedding of $K$ into $H_{F}$ if and only if no prime of $F$ which ramifies in $H_{F}$ splits in $K$.

Proposition 2.8. Let $F$ be a number field and let $K$ be a quadratic extension of $F$. Let $H_{F}$ be a quaternion algebra over $F$. Then $K$ splits $H_{F}$ if and only if there exists an $F$-embedding $K \hookrightarrow H_{F}$. 


\section{DIVISION QUATERNION ALGEBRAS OVER QUADRATIC NUMBER FIELDS}

In this section we collect some results which will be used often in the rest of this paper. The following lemma appears in the nice book of Alsina and Bayer ([3, Lemma 1.21]).

LEMMA 3.1. Let $p$ and $q$ be two primes, and let $H_{\mathbb{Q}}(p, q)$ be a quaternion algebra of discriminant $D_{H}$.

(i) if $p \equiv q \equiv 3(\bmod 4)$ and $\left(\frac{q}{p}\right) \neq 1$, then $D_{H}=2 p$;

(ii) if $q=2$ and $p \equiv 3(\bmod 8)$, then $D_{H}=p q=2 p$;

(iii) if $p$ or $q \equiv 1(\bmod 4)$, with $p \neq q$ and $\left(\frac{p}{q}\right)=-1$, then $D_{H}=p q$.

The following results were proved in our paper [1].

Proposition 3.2. Let $p$ and $q$ be two distinct odd primes, with $p$ or $q \equiv 1$ $(\bmod 4)$ and $\left(\frac{p}{q}\right)=-1$. Let $K=\mathbb{Q}(\sqrt{d})$ and let $\Delta_{K}$ be the discriminant of $K$. Then the quaternion algebra $H_{\mathbb{Q}(\sqrt{d})}(p, q)$ is a division algebra if and only if $\left(\frac{\Delta_{K}}{p}\right)=1$ or $\left(\frac{\Delta_{K}}{q}\right)=1$.

Proposition 3.3. Let $p$ be an odd prime, with $p \equiv 3(\bmod 8)$. Let $K=\mathbb{Q}(\sqrt{d})$ and let $\Delta_{K}$ be the discriminant of $K$. Then $H_{\mathbb{Q}(\sqrt{d})}(p, 2)$ is a division algebra if and only if $\left(\frac{\Delta_{K}}{p}\right)=1$ or $d \equiv 1(\bmod 8)$.

Proposition 3.4. Let $p$ and $q$ be two odd prime integers, with $p \equiv q \equiv 3$ $(\bmod 4)$ and $\left(\frac{q}{p}\right) \neq 1$. Let $K=\mathbb{Q}(\sqrt{d})$ and let $\Delta_{K}$ be the discriminant of $K$. Then the quaternion algebra $H_{\mathbb{Q}(\sqrt{d})}(p, q)$ is a division algebra if and only if $\left(\frac{\Delta_{K}}{p}\right)=1$ or $d \equiv 1(\bmod 8)$.

Lemma 3.5. Let $p \equiv 5(\bmod 8)$ be a prime integer. Then the discriminant of the quaternion algebra $H_{\mathbb{Q}}(p, 2)$ is equal to $2 p$, and therefore $H_{\mathbb{Q}}(p, 2)$ is a division algebra.

\section{Division QUATERnion ALGEBRAS OVER BIQUADRATIC NUMBER FIELDS}

Let us start with a proposition that will be useful in this section.

Proposition 4.1. Let $a$ and $b$ be distinct nonzero integers and let $p$ be an odd prime.

(i) if $a$ and $b$ are quadratic residues modulo $p$, then $\operatorname{lcm}(a, b) / \operatorname{gcd}(a, b)$ is also a quadratic residue modulo $p$;

(ii) if $a$ and $\operatorname{lcm}(a, b) / \operatorname{gcd}(a, b)$ are quadratic residues modulo $p$, then $b$ is a quadratic residue modulo $p$.

Proof. Since $a$ and $b$ are both quadratic residues modulo $p$ it follows that $\left(\frac{a b}{p}\right)=1$. Let $c=\frac{\operatorname{lcm}(a, b)}{\operatorname{gcd}(a, b)}=\frac{a b}{\operatorname{gcd}(a, b)^{2}}$. We have now $\left(\frac{\operatorname{gcd}(a, b)^{2}}{p}\right)=1$ and 
$\left(\frac{a b}{p}\right)=\left(\frac{\operatorname{gcd}(a, b)^{2}}{p}\right)\left(\frac{c}{p}\right)=1$. Therefore $\left(\frac{c}{p}\right)=1$, so $\frac{\operatorname{lcm}(a, b)}{\operatorname{gcd}(a, b)}$ is a quadratic residue modulo $p$.

The proof of the second case is similar to the proof of the first case.

Let $K / L$ be an extension of number fields. If a quaternion algebra $H_{L}(p, q)$ splits, then the quaternion algebra $H_{K}(p, q)$ splits as well. If the quaternion algebra $H_{L}(p, q)$ is a division algebra then the quaternion algebra $H_{K}(p, q)$ could still be a division algebra or else split.

We consider now a division quaternion algebra $H_{\mathbb{Q}\left(\sqrt{d_{1}}\right)}(p, q)$ over a base field $L=\mathbb{Q}\left(\sqrt{d_{1}}\right)$, and try to find some conditions which guarantee that it is still a division algebra over the biquadratic field $K=\mathbb{Q}\left(\sqrt{d_{1}}, \sqrt{d_{2}}\right)$.

Proposition 4.2. Let $d_{1}$ and $d_{2}$ be distinct squarefree integers not equal to one. Let $p$ and $q$ be distinct odd prime integers such that $\left(\frac{p}{q}\right)=-1$, and $p$ or $q$ is congruent to one modulo 4 . Let $K=\mathbb{Q}\left(\sqrt{d_{1}}, \sqrt{d_{2}}\right)$ and let $K_{i}=\mathbb{Q}\left(\sqrt{d_{i}}\right)$ $(i=1,2)$, with discriminant $\Delta_{K_{i}}$. Then the quaternion algebra $H_{K}(p, q)$ is a division algebra if and only if $\left(\frac{\Delta_{K_{1}}}{p}\right)=\left(\frac{\Delta_{K_{2}}}{p}\right)=1$ or $\left(\frac{\Delta_{K_{1}}}{q}\right)=\left(\frac{\Delta_{K_{2}}}{q}\right)=1$.

Proof. Let us assume that $H_{K}(p, q)$ is a division algebra. Hence the quaternion algebras $H_{\mathbb{Q}}(p, q), H_{K_{1}}(p, q), H_{K_{2}}(p, q)$ must all be division algebras.

Let $K_{3}=\mathbb{Q}\left(\sqrt{d_{3}}\right)$ be the third quadratic subfield of $K$, where $d_{3}=$ $\frac{\operatorname{lcm}\left(d_{1}, d_{2}\right)}{\operatorname{gcd}\left(d_{1}, d_{2}\right)}$, with discriminant $\Delta_{K_{3}}$. Since $H_{K}(p, q)$ is a division algebra, it follows that $H_{K_{3}}(p, q)$ must be a division algebra as well. Proposition 3.2 tells us that one of the following conditions must be verified:

(i) $\left(\frac{\Delta_{K_{1}}}{p}\right)=\left(\frac{\Delta_{K_{2}}}{p}\right)=\left(\frac{\Delta_{K_{3}}}{p}\right)=1$;

(ii) $\left(\frac{\Delta_{K_{1}}}{q}\right)=\left(\frac{\Delta_{K_{2}}}{q}\right)=\left(\frac{\Delta_{K_{3}}}{q}\right)=1$;

(iii) There are $i, j \in\{1,2,3\}, i \neq j$ such that $\left(\frac{\Delta_{K_{i}}}{p}\right)=\left(\frac{\Delta_{K_{j}}}{p}\right)=1$ and there is $l \in\{1,2,3\}, l \neq i, l \neq j$ such that $\left(\frac{\Delta_{K_{l}}}{q}\right)=1$. Then $d_{i}$ and $d_{j}$ are quadratic residues modulo $p$. From Proposition 4.1 it follows that $d_{1}, d_{2}, d_{3}$ are quadratic residues modulo $p$, so $\left(\frac{\Delta_{K_{1}}}{p}\right)=\left(\frac{\Delta_{K_{2}}}{p}\right)=$ $\left(\frac{\Delta_{K_{3}}}{p}\right)=1$;

(iv) There are $i, j \in\{1,2,3\}, i \neq j$ such that $\left(\frac{\Delta_{K_{i}}}{q}\right)=\left(\frac{\Delta_{K_{j}}}{q}\right)=1$ and there is $l \in\{1,2,3\}, l \neq i, l \neq j$ such that $\left(\frac{\Delta_{K_{l}}}{p}\right)=1$. From Proposition 4.1 it follows that $\left(\frac{\Delta_{K_{1}}}{q}\right)=\left(\frac{\Delta_{K_{2}}}{q}\right)=\left(\frac{\Delta_{K_{3}}}{q}\right)=1$.

Conversely, let us assume that $\left(\frac{\Delta_{K_{1}}}{p}\right)=\left(\frac{\Delta_{K_{2}}}{p}\right)=1$. By Proposition 4.1 it follows that $\left(\frac{\Delta_{K_{3}}}{p}\right)=1$. By Theorem 2.1(ii) it follows that $p$ splits in each $\mathcal{O}_{K_{i}}$. According to Theorem 2.2, $p$ must split in $\mathcal{O}_{K}$ as well. Since $\left(\frac{\Delta_{K_{1}}}{p}\right)=1$, Proposition 3.2 tells us that $H_{K_{1}}(p, q)$ must be a division algebra, and that $p$ 
must ramify in $H_{K_{1}}(p, q)$. By Theorem 2.7, Proposition 2.8 and Proposition 2.6 , the quaternion algebra $H_{K}(p, q)$ must be a division algebra.

If $\left(\frac{\Delta_{K_{1}}}{q}\right)=\left(\frac{\Delta_{K_{2}}}{q}\right)=\left(\frac{\Delta_{K_{3}}}{q}\right)=1$, the same argument shows that $H_{K}(p, q)$ must be a division algebra.

Proposition 4.3. Let $d_{1}$ and $d_{2}$ be distinct squarefree integers not equal to one. Let $p$ an odd prime integer, such that $p \equiv 3(\bmod 8)$. Let $K=$ $\mathbb{Q}\left(\sqrt{d_{1}}, \sqrt{d_{2}}\right)$, and let $K_{i}=\mathbb{Q}\left(\sqrt{d_{i}}\right)(i=1,2)$, with discriminant $\Delta_{K_{i}}$. Then the quaternion algebra $H_{K}(p, 2)$ is a division algebra if and only if $\left(\frac{\Delta_{K_{1}}}{p}\right)=$ $\left(\frac{\Delta_{K_{2}}}{p}\right)=1$ or $d_{1}, d_{2} \equiv 1(\bmod 8)$.

Proof. Let us assume that $H_{K}(p, 2)$ is a division algebra. Then the quaternion algebras $H_{\mathbb{Q}}(p, 2), H_{K_{1}}(p, 2), H_{K_{2}}(p, 2)$ are division algebras as well.

Let $K_{3}=\mathbb{Q}\left(\sqrt{d_{3}}\right)$ be the third quadratic subfield of $K$, where $d_{3}=$ $\frac{\operatorname{lcm}\left(d_{1}, d_{2}\right)}{\operatorname{gcd}\left(d_{1}, d_{2}\right)}$, with discriminant $\Delta_{K_{3}}$. Since $H_{K}(p, q)$ is a division algebra, it follows that $H_{K_{3}}(p, q)$ must be a division algebra as well.

Let $D_{H}$ be the discriminant of the quaternion algebra $H_{\mathbb{Q}}(p, q)$. According to the hypothesis and to Lemma 3.1(ii), we must have $D_{H}=2 p$. Proposition 3.3 tells us that one of the following conditions must be verified:

(i) $\left(\frac{\Delta_{K_{1}}}{p}\right)=\left(\frac{\Delta_{K_{2}}}{p}\right)=\left(\frac{\Delta_{K_{3}}}{p}\right)=1$;

(ii) $d_{1}, d_{2}, d_{3} \equiv 1(\bmod 8)$;

(iii) There are $i, j \in\{1,2,3\}, i \neq j$ such that $\left(\frac{\Delta_{K_{i}}}{p}\right)=\left(\frac{\Delta_{K_{j}}}{p}\right)=1$ and there is $l \in\{1,2,3\}, l \neq i, l \neq j$ such that $d_{l} \equiv 1(\bmod 8)$. Then, $d_{i}$ and $d_{j}$ are quadratic residues modulo $p$. From Proposition 4.1 it follows that $d_{1}, d_{2}, d_{3}$ are quadratic residues modulo $p$, so $\left(\frac{\Delta_{K_{1}}}{p}\right)=\left(\frac{\Delta_{K_{2}}}{p}\right)=$ $\left(\frac{\Delta_{K_{3}}}{p}\right)=1$

(iv) There are $i, j \in\{1,2,3\}, i \neq j$ such that $d_{i}, d_{j} \equiv 1(\bmod 8)$ and there is $l \in\{1,2,3\}, l \neq i, l \neq j$ such that $\left(\frac{\Delta_{K_{l}}}{p}\right)=1$. Since $d_{3}=\frac{\operatorname{lcm}\left(d_{1}, d_{2}\right)}{\operatorname{gcd}\left(d_{1}, d_{2}\right)}$ it follows that $d_{1}, d_{2}, d_{3} \equiv 1(\bmod 8)$.

Let us now prove the converse. If $\left(\frac{\Delta_{K_{1}}}{p}\right)=\left(\frac{\Delta_{K_{2}}}{p}\right)=1$ the argument is the same used in the proof of sufficiency of Proposition 4.2.

If $d_{1}, d_{2} \equiv 1(\bmod 8)$, it follows easily that $d_{3} \equiv 1(\bmod 8)$. According to Proposition 3.3, the algebras $H_{K_{1}}(p, 2), H_{K_{2}}(p, 2)$ and $H_{K_{3}}(p, 2)$ are all division algebras and 2 ramifies there. From Theorem 2.1 it follows that 2 splits completely in $K_{1}, K_{2}$ and $K_{3}$, and from Theorem 2.2 we can conclude that 2 splits completely in $K$. Finally, from Theorem 2.7, Proposition 2.8 and Proposition 2.6, it follows now that $H_{K}(p, 2)$ is a division algebra.

Proposition 4.4. Let $d_{1}$ and $d_{2}$ be distinct squarefree integers not equal to one. Let $p$ and $q$ be distinct odd prime integers, with $p \equiv q \equiv 3(\bmod 4)$ 
and $\left(\frac{q}{p}\right)=-1$. Let $K=\mathbb{Q}\left(\sqrt{d_{1}}, \sqrt{d_{2}}\right)$, and let $K_{i}=\mathbb{Q}\left(\sqrt{d_{i}}\right)(i=1,2)$, with discriminant $\Delta_{K_{i}}$. Then the quaternion algebra $H_{K}(p, q)$ is a division algebra if and only if $\left(\frac{\Delta_{K_{1}}}{p}\right)=\left(\frac{\Delta_{K_{2}}}{p}\right)=1$ or $d_{1}, d_{2} \equiv 1(\bmod 8)$.

Proof. The proof is similar to the proof of Proposition 4.3.

Proposition 4.5. Let $d_{1}$ and $d_{2}$ be distinct squarefree integers not equal to one. Let $p$ an odd prime integer, such that $p \equiv 5(\bmod 8)$. Let $K=$ $\mathbb{Q}\left(\sqrt{d_{1}}, \sqrt{d_{2}}\right)$, and let $K_{i}=\mathbb{Q}\left(\sqrt{d_{i}}\right)(i=1,2)$, with discriminant $\Delta_{K_{i}}$. Then the quaternion algebra $H_{K}(p, 2)$ is a division algebra if and only if $\left(\frac{\Delta_{K_{1}}}{p}\right)=$ $\left(\frac{\Delta_{K_{2}}}{p}\right)=1$ or $d_{1}, d_{2} \equiv 1(\bmod 8)$.

Proof. The proof is similar to the proof of Proposition 4.3.

After gluing the last three proposition together, we obtain the first significant result of this paper.

Theorem 4.6 (Classification over biquadratic fields). Let $d_{1}$ and $d_{2}$ be distinct squarefree integers not equal to one. Let $K=\mathbb{Q}\left(\sqrt{d_{1}}, \sqrt{d_{2}}\right)$, and let $K_{i}=\mathbb{Q}\left(\sqrt{d_{i}}\right)(i=1,2)$ with discriminant $\Delta_{K_{i}}$. Let $p$ and $q$ be two positive primes. Then the quaternion algebra $H_{K}(p, q)$ is a division algebra if and only if one of the following conditions holds:

(i) $p$ and $q$ are odd and distinct, and

$$
\left(\frac{p}{q}\right)=-1 \text {, and }
$$

$p \equiv 1(\bmod 4)$ or $q \equiv 1(\bmod 4)$, and

$\left(\frac{\Delta_{K_{1}}}{p}\right)=\left(\frac{\Delta_{K_{2}}}{p}\right)=1$ or $\left(\frac{\Delta_{K_{1}}}{q}\right)=\left(\frac{\Delta_{K_{2}}}{q}\right)=1$;

(ii) $q=2$, and

$p \equiv 3(\bmod 8)$ or $p \equiv 5(\bmod 8)$, and

either $\left(\frac{\Delta_{K_{1}}}{p}\right)=\left(\frac{\Delta_{K_{2}}}{p}\right)=1$ or $d_{1}, d_{2} \equiv 1(\bmod 8)$;

(iii) $p$ and $q$ are odd, with $p \equiv q \equiv 3(\bmod 4)$, and

- $\left(\frac{q}{p}\right) \neq 1$, and

- either $\left(\frac{\Delta_{K_{1}}}{p}\right)=\left(\frac{\Delta_{K_{2}}}{p}\right)=1$ or $d_{1}, d_{2} \equiv 1(\bmod 8)$;

or

- $\left(\frac{p}{q}\right) \neq 1$, and

- either $\left(\frac{\Delta_{K_{1}}}{q}\right)=\left(\frac{\Delta_{K_{2}}}{q}\right)=1$ or $d_{1}, d_{2} \equiv 1(\bmod 8)$.

Proof. The theorem follows easily from the four propositions above, after taking into account Lemma 3.1 and Lemma 3.4.

Let us point out that in the first case of the classification theorem we have a manifest symmetry, i.e. by Legendre's statement of the quadratic reciprocity law $p$ is a quadratic residue modulo $q$ if and only if $q$ is a quadratic residue mod $p$, while in the third case the setting is asymmetrical, i.e., again by Legendre's 
statement of the quadratic reciprocity law, $p$ is a quadratic residue modulo $q$ if and only if $q$ is not a quadratic residue modulo $p$.

\section{Division quaternion Algebras OVER THE COMPOSITE OF $n$ QUADRATIC FIELDS}

In this section we show how to apply the technique of proof shown in the previous section to classify quaternion algebras over the composite of $n$ quadratic fields.

Let $d_{1}, \ldots, d_{n}$ be distinct integers not equal to one. We say that $d_{1}, \ldots, d_{n}$ are multiplicatively independent over $\mathbb{Q}$ if the equation $d_{1}^{x_{1}} \cdots d_{n}^{x_{n}}=1$ in the indeterminates $x_{1}, \ldots, x_{n}$ admits no rational solution distinct from $(0, \ldots, 0)$.

When $d_{1}, \ldots, d_{n}$ are distinct squarefree integers not equal to one it is easily seen that $d_{1}, \ldots, d_{n}$ are multiplicatively independent over $\mathbb{Q}$ if, once $d_{1}, \ldots, d_{n}$ are written as products of prime powers $d_{i}=p_{1}^{e_{1, i}} \cdots p_{k}^{e_{k, i}}$ then the vectors of exponents $\left(e_{1, i} \ldots e_{k, i}\right)$ are linearly independent mod 2 . In this case it can be shown $\left[16\right.$, p. 267-268] that $K=\mathbb{Q}\left(\sqrt{d_{1}}, \ldots, \sqrt{d_{n}}\right)$ is a Galois extension of $\mathbb{Q}$ with Galois group $\underbrace{\mathbb{Z}_{2} \times \cdots \times \mathbb{Z}_{2}}_{n \text { times }}$.

Let us start with the smallest case, that is with $n=3$. Hence we take a quaternion algebra $H_{\mathbb{Q}\left(\sqrt{d_{1}}, \sqrt{d_{2}}\right)}(p, q)$ over the base field $L=\mathbb{Q}\left(\sqrt{d_{1}}, \sqrt{d_{2}}\right)$, and we try to find some conditions which guarantee that it is still a division algebra over the field $K=\mathbb{Q}\left(\sqrt{d_{1}}, \sqrt{d_{2}}, \sqrt{d_{3}}\right)$.

Let us recall the following well known theorem ([26, p.360]).

THEOREM 5.1. Suppose that $p$ is a prime of $\mathbb{Q}$ which splits completely in each of two fields $F_{1}$ and $F_{2}$. Then $p$ splits completely in the composite field $F_{1} F_{2}$.

As a consequence, if $p$ splits completely in a field $F$, then $p$ also splits completely in the minimal normal extension of $\mathbb{Q}$ containing $F$. We obtain the following result.

Proposition 5.2. Let $d_{1}, d_{2}$ and $d_{3}$ be distinct squarefree integers not equal to one, multiplicatively independent over $\mathbb{Q}$. Let $p$ and $q$ be distinct odd prime integers such that $\left(\frac{p}{q}\right)=-1, p$ does not divide $d_{i}$ and $q$ does not divide $d_{i},(i=1,2,3)$ and $p$ or $q$ is congruent to one modulo 4 . Let $K=\mathbb{Q}\left(\sqrt{d_{1}}, \sqrt{d_{2}}, \sqrt{d_{3}}\right)$ and let $K_{i}=\mathbb{Q}\left(\sqrt{d_{i}}\right)(i=1,2,3)$, with discriminant $\Delta_{K_{i}}$. Then the quaternion algebra $H_{K}(p, q)$ is a division algebra if and only if $\left(\frac{\Delta_{K_{1}}}{p}\right)=\left(\frac{\Delta_{K_{2}}}{p}\right)=\left(\frac{\Delta_{K_{3}}}{p}\right)=1$ or $\left(\frac{\Delta_{K_{1}}}{q}\right)=\left(\frac{\Delta_{K_{2}}}{q}\right)=\left(\frac{\Delta_{K_{3}}}{q}\right)=1$.

ProOF. If $H_{K}(p, q)$ is a division algebra, then, according to Proposition 4.2:

- $H_{\mathbb{Q}\left(\sqrt{d_{1}}, \sqrt{d_{2}}\right)}(p, q)$ is a division algebra, and this is equivalent to $\left(\frac{\Delta_{K_{1}}}{p}\right)=\left(\frac{\Delta_{K_{2}}}{p}\right)=1$ or $\left(\frac{\Delta_{K_{1}}}{q}\right)=\left(\frac{\Delta_{K_{2}}}{q}\right)=1$; 
- $H_{\mathbb{Q}\left(\sqrt{d_{1}}, \sqrt{d_{3}}\right)}(p, q)$ is a division algebra, and this is equivalent to $\left(\frac{\Delta_{K_{1}}}{p}\right)=\left(\frac{\Delta_{K_{3}}}{p}\right)=1$ or $\left(\frac{\Delta_{K_{1}}}{q}\right)=\left(\frac{\Delta_{K_{3}}}{q}\right)=1$;

- $H_{\mathbb{Q}\left(\sqrt{d_{2}}, \sqrt{d_{3}}\right)}(p, q)$ is a division algebra, and this is equivalent to $\left(\frac{\Delta_{K_{2}}}{p}\right)=\left(\frac{\Delta_{K_{3}}}{p}\right)=1$ or $\left(\frac{\Delta_{K_{2}}}{q}\right)=\left(\frac{\Delta_{K_{3}}}{q}\right)=1$.

Therefore, one of the following conditions must be satisfied:

(i) $\left(\frac{\Delta_{K_{1}}}{p}\right)=\left(\frac{\Delta_{K_{2}}}{p}\right)=\left(\frac{\Delta_{K_{3}}}{p}\right)=1$;

(ii) $\left(\frac{\Delta_{K_{1}}}{q}\right)=\left(\frac{\Delta_{K_{2}}}{q}\right)=\left(\frac{\Delta_{K_{3}}}{q}\right)=1$;

(iii) there are $i, j \in\{1,2,3\}, i \neq j$ such that $\left(\frac{\Delta_{K_{i}}}{p}\right)=\left(\frac{\Delta_{K_{j}}}{p}\right)=1$ and there is $l \in\{1,2,3\}, l \neq i, l \neq j$ such that $\left(\frac{\Delta_{K_{i}}}{q}\right)=\left(\frac{\Delta_{K_{l}}}{q}\right)=1$ and $\left(\frac{\Delta_{K_{j}}}{q}\right)=\left(\frac{\Delta_{K_{l}}}{q}\right)=1$. It results that $\left(\frac{\Delta_{K_{1}}}{q}\right)=\left(\frac{\Delta_{K_{2}}}{q}\right)=\left(\frac{\Delta_{K_{3}}}{q}\right)=1 ;$

(iv) there are $i, j, k \in\{1,2,3\}, i \neq j \neq k \neq i$ such that $\left(\frac{\Delta_{K_{i}}}{p}\right)=\left(\frac{\Delta_{K_{j}}}{p}\right)=1$ and $\left(\frac{\Delta_{K_{j}}}{p}\right)=\left(\frac{\Delta_{K_{l}}}{p}\right)=1$. It results that $\left(\frac{\Delta_{K_{1}}}{p}\right)=\left(\frac{\Delta_{K_{2}}}{p}\right)=\left(\frac{\Delta_{K_{3}}}{p}\right)=1$.

Conversely, let us suppose that $\left(\frac{\Delta_{K_{1}}}{p}\right)=\left(\frac{\Delta_{K_{2}}}{p}\right)=\left(\frac{\Delta_{K_{3}}}{p}\right)=1$. Since $\left(\frac{\Delta_{K_{1}}}{p}\right)=\left(\frac{\Delta_{K_{2}}}{p}\right)=1$, from Theorem 2.2 it follows that $p$ splits in $\mathbb{Q}\left(\sqrt{d_{1}}, \sqrt{d_{2}}\right)$. Since $\left(\frac{\Delta_{K_{3}}}{p}\right)=1$, from Theorem 2.1 it follows that $p$ splits in $\mathbb{Q}\left(\sqrt{d_{3}}\right)$. According to Theorem 5.1, $p$ must split in $K$. According to Proposition 4.2, $H_{\mathbb{Q}\left(\sqrt{d_{1}}, \sqrt{d_{2}}\right)}(p, q)$ is a division algebra and $p$ ramifies in $H_{\mathbb{Q}\left(\sqrt{d_{1}}, \sqrt{d_{2}}\right)}(p, q)$. By Theorem 2.7, Proposition 2.8 and Proposition 2.6, the quaternion algebra $H_{K}(p, q)$ must be a division algebra.

If $\left(\frac{\Delta_{K_{1}}}{q}\right)=\left(\frac{\Delta_{K_{2}}}{q}\right)=\left(\frac{\Delta_{K_{3}}}{q}\right)=1$, the same argument shows that $H_{K}(p, q)$ is a division algebra.

We can generalize now Proposition 4.2 and Proposition 5.2.

Proposition 5.3. Let $n$ be a positive integer, $n \geq 2$ and let $d_{1}, d_{2}, \ldots, d_{n}$ be distinct squarefree integers not equal to one, multiplicatively independent over $\mathbb{Q}$. Let $p$ and $q$ be distinct odd prime integers such that $\left(\frac{p}{q}\right)=-1, p$ does not divide $d_{i}$ and $q$ does not divide $d_{i}(i=1, \ldots, n)$, and $p$ or $q$ is congruent to one modulo 4 . Let $K=\mathbb{Q}\left(\sqrt{d_{1}}, \sqrt{d_{2}}, \ldots, \sqrt{d_{n}}\right)$ and let $K_{i}=\mathbb{Q}\left(\sqrt{d_{i}}\right)(i=$ $1, \ldots, n)$, with discriminant $\Delta_{K_{i}}$. Then the quaternion algebra $H_{K}(p, q)$ is a division algebra if and only if $\left(\frac{\Delta_{K_{1}}}{p}\right)=\left(\frac{\Delta_{K_{2}}}{p}\right)=\cdots=\left(\frac{\Delta_{K_{n}}}{p}\right)=1$ or $\left(\frac{\Delta_{K_{1}}}{q}\right)=\left(\frac{\Delta_{K_{2}}}{q}\right)=\cdots=\left(\frac{\Delta_{K_{n}}}{q}\right)=1$.

Proof. The proof is by mathematical induction over $n$, for $n>2$. The inductive step is based on the same argument that we used to go from Proposition 4.2 to Proposition 5.2.

Proposition 5.4. Let $d_{1}, d_{2}$ and $d_{3}$ be distinct squarefree integers not equal to one, multiplicatively independent over $\mathbb{Q}$. Let $p$ be an odd prime 
integer such that $p$ does not divide $d_{i}, i=1,2,3, p \equiv 3(\bmod 8)$. Let $K=\mathbb{Q}\left(\sqrt{d_{1}}, \sqrt{d_{2}}, \sqrt{d_{3}}\right)$ and let $K_{i}=\mathbb{Q}\left(\sqrt{d_{i}}\right)(i=1,2,3)$, with discriminant $\Delta_{K_{i}}$. Then the quaternion algebra $H_{K}(p, 2)$ is a division algebra if and only if $\left(\frac{\Delta_{K_{1}}}{p}\right)=\left(\frac{\Delta_{K_{2}}}{p}\right)=\left(\frac{\Delta_{K_{3}}}{p}\right)=1$ or $d_{1}, d_{2}, d_{3} \equiv 1(\bmod 8)$.

Proof. If $H_{K}(p, 2)$ is a division algebra, then $H_{\mathbb{Q}\left(\sqrt{d_{1}}, \sqrt{d_{2}}\right)}(p, q)$ is a division algebra and this is equivalent with $\left(\frac{\Delta_{K_{1}}}{p}\right)=\left(\frac{\Delta_{K_{2}}}{p}\right)=1$ or $d_{1}, d_{2} \equiv 1$ $(\bmod 8) ; H_{\mathbb{Q}\left(\sqrt{d_{1}}, \sqrt{d_{3}}\right)}(p, q)$ is a division algebra and this is equivalent with $\left(\frac{\Delta_{K_{1}}}{p}\right)=\left(\frac{\Delta_{K_{3}}}{p}\right)=1$ or $d_{1}, d_{3} \equiv 1(\bmod 8) ; H_{\mathbb{Q}\left(\sqrt{d_{2}}, \sqrt{d_{3}}\right)}(p, q)$ is a division algebra and this is equivalent with $\left(\frac{\Delta_{K_{2}}}{p}\right)=\left(\frac{\Delta_{K_{3}}}{p}\right)=1$ or $d_{2}, d_{3} \equiv 1(\bmod 8)$ (according to Proposition 4.3). Considering these we can have one of the following cases:

(i) $\left(\frac{\Delta_{K_{1}}}{p}\right)=\left(\frac{\Delta_{K_{2}}}{p}\right)=\left(\frac{\Delta_{K_{3}}}{p}\right)=1$;

(ii) $d_{1}, d_{2}, d_{3} \equiv 1(\bmod 8)$;

(iii) there are $i, j \in\{1,2,3\}, i \neq j$ such that $\left(\frac{\Delta_{K_{i}}}{p}\right)=\left(\frac{\Delta_{K_{j}}}{p}\right)=1$ and there is $l \in\{1,2,3\}, l \neq i, l \neq j$ such that $d_{i}, d_{l} \equiv 1(\bmod 8)$ and $d_{j}, d_{l} \equiv 1$ $(\bmod 8)$. It results that $d_{1}, d_{2}, d_{3} \equiv 1(\bmod 8)$

(iv) there are $i, j, k \in\{1,2,3\}, i \neq j \neq k \neq i$ such that $\left(\frac{\Delta_{K_{i}}}{p}\right)=\left(\frac{\Delta_{K_{j}}}{p}\right)=$ 1 and $\left(\frac{\Delta_{K_{i}}}{p}\right)=\left(\frac{\Delta_{K_{l}}}{p}\right)=1$ and $d_{j}, d_{l} \equiv 1(\bmod 8)$. It results that $\left(\frac{\Delta_{K_{1}}}{p}\right)=\left(\frac{\Delta_{K_{2}}}{p}\right)=\left(\frac{\Delta_{K_{3}}}{p}\right)=1$.

Conversely, if $\left(\frac{\Delta_{K_{1}}}{p}\right)=\left(\frac{\Delta_{K_{2}}}{p}\right)=\left(\frac{\Delta_{K_{3}}}{p}\right)=1$, then the argument is the same used in the proof of suffciency of Proposition 5.2.

If $d_{1}, d_{2}, d_{3} \equiv 1(\bmod 8)$, applying to Proposition 4.3 , it results that the quaternion algebra $H_{\mathbb{Q}\left(\sqrt{d_{1}}, \sqrt{d_{2}}\right)}(p, q)$ is a division algebras and 2 ramifies this algebra. According to Theorem 2.1 it follows that 2 splits completely in $K_{1}, K_{2}$ and $K_{3}$. From Theorem 2.2 it follows that 2 splits completely in $\mathbb{Q}\left(\sqrt{d_{1}}, \sqrt{d_{2}}\right)$. Using this and the fact that 2 splits completely in $K_{3}$, applying Proposition 5.1, we conclude that 2 splits completely in $K$. According to Theorem 2.7, Proposition 2.8 and Proposition 2.6, it follows now that $H_{K}(p, 2)$ is a division algebra.

The following two propositions can be proven in a similar way and we present them without proofs.

Proposition 5.5. Let $d_{1}, d_{2}$ and $d_{3}$ be distinct squarefree integers not equal to one, multiplicatively independent over $\mathbb{Q}$. Let $p, q$ be two odd prime integers such that $p$ does not divide $d_{i}, i=1,2,3, p \equiv q \equiv 3(\bmod 4),\left(\frac{q}{p}\right) \neq 1$. Let $K=\mathbb{Q}\left(\sqrt{d_{1}}, \sqrt{d_{2}}, \sqrt{d_{3}}\right)$ and let $K_{i}=\mathbb{Q}\left(\sqrt{d_{i}}\right)(i=1,2,3)$, with discriminant $\Delta_{K_{i}}$. Then the quaternion algebra $H_{K}(p, q)$ is a division algebra if and only if $\left(\frac{\Delta_{K_{1}}}{p}\right)=\left(\frac{\Delta_{K_{2}}}{p}\right)=\left(\frac{\Delta_{K_{3}}}{p}\right)=1$ or $d_{1}, d_{2}, d_{3} \equiv 1(\bmod 8)$. 
Proposition 5.6. Let $d_{1}, d_{2}$ and $d_{3}$ be distinct squarefree integers not equal to one, multiplicatively independent over $\mathbb{Q}$. Let $p$ be an odd prime integer such that $p$ does not divide $d_{i}, i=1,2,3, p \equiv 5(\bmod 8)$. Let $K=\mathbb{Q}\left(\sqrt{d_{1}}, \sqrt{d_{2}}, \sqrt{d_{3}}\right)$ and let $K_{i}=\mathbb{Q}\left(\sqrt{d_{i}}\right)(i=1,2,3)$, with discriminant $\Delta_{K_{i}}$. Then the quaternion algebra $H_{K}(p, 2)$ is a division algebra if and only if $\left(\frac{\Delta_{K_{1}}}{p}\right)=\left(\frac{\Delta_{K_{2}}}{p}\right)=\left(\frac{\Delta_{K_{3}}}{p}\right)=1$ or $d_{1}, d_{2}, d_{3} \equiv 1(\bmod 8)$.

If we take into account the results obtained in Proposition 5.2, Proposition 5.4, Proposition 5.5, Proposition 5.6, we obtain the following classification theorem.

Theorem 5.7 (Classification over the composite of three quadratic fields). Let $d_{1}, d_{2}, d_{3}$ be distinct squarefree integers not equal to one, multiplicatively independent over $\mathbb{Q}$. Let $K=\mathbb{Q}\left(\sqrt{d_{1}}, \sqrt{d_{2}}, \sqrt{d_{3}}\right)$, and let $K_{i}=\mathbb{Q}\left(\sqrt{d_{i}}\right)(i=$ $1,2,3)$, with discriminant $\Delta_{K_{i}}$. Let $p$ and $q$ be two positive primes. Then the quaternion algebra $H_{K}(p, q)$ is a division algebra if and only if one of the following conditions holds:

(i) $p$ and $q$ are odd and distinct, and $\left(\frac{p}{q}\right)=-1$, and

$p \equiv 1(\bmod 4)$ or $q \equiv 1(\bmod 4)$, and

$\left(\frac{\Delta_{K_{1}}}{p}\right)=\left(\frac{\Delta_{K_{2}}}{p}\right)=\left(\frac{\Delta_{K_{3}}}{p}\right)=1$ or $\left(\frac{\Delta_{K_{1}}}{q}\right)=\left(\frac{\Delta_{K_{2}}}{q}\right)=\left(\frac{\Delta_{K_{3}}}{q}\right)=1 ;$

(ii) $q=2$, and

$p \equiv 3(\bmod 8)$ or $p \equiv 5(\bmod 8)$, and

either $\left(\frac{\Delta_{K_{1}}}{p}\right)=\left(\frac{\Delta_{K_{2}}}{p}\right)=\left(\frac{\Delta_{K_{3}}}{p}\right)=1$ or $d_{1}, d_{2}, d_{3} \equiv 1(\bmod 8)$;

(iii) $p$ and $q$ are odd, with $p \equiv q \equiv 3(\bmod 4)$, and

- $\left(\frac{q}{p}\right) \neq 1$, and

- either $\left(\frac{\Delta_{K_{1}}}{p}\right)=\left(\frac{\Delta_{K_{2}}}{p}\right)=\left(\frac{\Delta_{K_{3}}}{p}\right)=1$ or $d_{1}, d_{2}, d_{3} \equiv 1(\bmod 8)$;

or

- $\left(\frac{p}{q}\right) \neq 1$, and

- either $\left(\frac{\Delta_{K_{1}}}{q}\right)=\left(\frac{\Delta_{K_{2}}}{q}\right)=\left(\frac{\Delta_{K_{3}}}{q}\right)=1$ or $d_{1}, d_{2}, d_{3} \equiv 1(\bmod 8)$.

Proof. The theorem follows easily from the propositions above, after taking into account Lemma 3.1 and Lemma 3.4.

We have now all the ingredients needed to prove our Main Theorem, stated in Section 1.

Proof of the Main Theorem. By mathematical induction over $n$, for $n \geq 2$, we can generalize Propositions 5.4, 5.5 and 5.6 to obtain the sought classification of the division quaternion algebras over the composite of $n$ quadratic fields. 


\section{FinAL REMARKS}

The task of deciding whether a quaternion algebra over a number field is a division algebra is computationally a feasible one, thanks to the facilities included in computational algebra packages like Magma ([14]), Pari, Sage, etc; however, different approaches lead to very different execution times.

A naive way to check if a quaternion algebra is a division algebra is to show that a norm equation has no solution, thanks to Proposition 2.5 and Proposition 2.6. The problem of determining whether a norm equation over an extension of number fields has a solution has been extensively investigated in the past, both over arbitrary and over specific extensions of number fields $([2,8,9,21])$. Algorithms included in Magma, Pari and Sage allow one to find out whether a norm equation has or not at least a solution, and, sometime, to find a sought solution. However in the general case this is not an easy task.

Let us consider first the apparently efficient approach based on Proposition 2.5. For this purpose we wrote two small functions in SAGE, release 8.1, to test the efficiency of this method, and we used them to test an increasing number of cases (100 - 1000 - 10000 - 100000) of quaternion algebras.

In order to construct the cases to check, we considered $r$ unordered couples $\{p, q\}$ of positive primes $p$ and $q$, for increasing values of $p$ and $q$ starting from 2 , and for $K=\mathbb{Q}\left(\sqrt{d_{1}}, \sqrt{d_{2}}\right)$, we took approximately an equal number $r$ of unordered couples $\left\{ \pm d_{1}, \pm d_{2}\right\}$ of squarefree integers, for increasing values of $d_{1}$ and $d_{2}$ starting from 1 , omitting the trivial cases which would give $K=\mathbb{Q}(\sqrt{t})$ or $K=\mathbb{Q}$.

We ran our tests on a $2.6 \mathrm{Ghz}$ - i7 quad core - mac mini (late 2012), equipped with $8 \mathrm{~GB}$ of RAM. The running time is shown in the second column of Table 1. The value "n.a" means that the computation was taking too long and hence we forced the termination of the program.

A different approach to check if a quaternion algebra is a division algebra, based on Proposition 2.3, is to show that the discriminant ideal of the algebra is not equal to the full ring of integers of the base field. Again we wrote two small functions in SAGE to test the efficiency of this approach. The running time is shown in the third column of Table 1.

\begin{tabular}{|l|l|l|l|}
\hline \# of algebras & Norm approach & Discriminant approach & Our approach \\
\hline 100 & 2501 & 1044 & 4 \\
1000 & 43329 & 9428 & 42 \\
10000 & n.a. & 97049 & 384 \\
100000 & n.a. & 1000279 & 3818 \\
\hline
\end{tabular}

TABLE 1. Running time in ms. required to test $H_{\mathbb{Q}\left(\sqrt{d_{1}}, \sqrt{d_{2}}\right)}(p, q)$.

The approach described in this paper does not require one to solve norm equations over relative extensions of number fields, neither to compute the 
discriminant of quaternion algebras defined over an arbitrary number field. In fact, all we need is to compute a few Legendre symbols and the discriminants of quadratic extensions of $\mathbb{Q}$ involved, which is a very easy task: indeed, for a nonzero square free integer $d$, the discriminant of the quadratic field $\mathbb{Q}(\sqrt{d})$ is $d$ if $d$ is congruent to 1 modulo 4 , otherwise $4 d$. We wrote two small functions in SAGE to test the efficiency of our approach. The running time is shown in the fourth column of Table 1.

ACKNOWLEDGEMENTS.

We want to thank David Leep and Adam Chapman for their interesting remarks, which allowed us to greatly improve the original paper. We want express our gratitude to the anonymous reviewers for their careful reading of the manuscript and for many valuable suggestions. Also, the second author wants to thank Professor Victor Alexandru, for the interesting discussions on this topic.

\section{REFERENCES}

[1] V. Acciaro, D. Savin, M. Taous and A. Zekhnini, On quaternion algebras that split over specific quadratic number fields, to appear in Ital. J. Pure Appl. Math.

[2] V. Acciaro, Solvability of norm equations over cyclic number fields of prime degree, Math. Comp. 65 (1996), 1663-1674.

[3] M. Alsina and P. Bayer, Quaternion orders, quadratic forms and shimura curves, American Mathematical Society, Providence, 2004.

[4] W. Bosma, J. Cannon and C. Playoust, The Magma algebra system. I. The user language, J. Symbolic Comput. 24 (1997), 235-265.

[5] A. Chapman, D. J. Grynkiewicz, E. Matzri, L. H. Rowen and U. Vishne, Kummer spaces in symbol algebras of prime degree, J. Pure Appl. Algebra 220 (2016), 33633371.

[6] A. Chapman, Symbol length of p-algebras of prime exponent, J. Algebra Appl. 16 (2017), 1750136, 9 pp.

[7] T. Chinburg and E. Friedman, An embedding theorem for quaternion algebras, J. London Math. Soc. (2) 60 (1999), 33-44.

[8] C. Fieker, A. Jurk and M. Pohst, On solving relative norm equations in algebraic number fields, Math. Comp. 66 (1997), 399-410.

[9] U. Fincke and M. Pohst, A Procedure for Determining Algebraic Integers of Given Norm, in: Computer Algebra. EUROCAL 1983. Lecture Notes in Computer Science, vol 162, eds: J. A. van Hulzen, Springer, Berlin, Heidelberg, 1983, 194-202.

[10] P. Gille and T. Szamuely, Central simple algebras and Galois cohomology, Cambridge University Press, Cambridge, 2006.

[11] D. Goldstein and M. Schacher, Norms in central simple algebras, Pacific J. Math. 292 (2018), 373-388.

[12] K. Ireland and M. Rosen, A classical introduction to modern number theory, Springer, New York, 1990.

[13] C. U. Jensen and N. Yui, Polynomials with $D_{p}$ as Galois group, J. Number Theory 15 (1982), 347-375.

[14] D. R. Kohel, Quaternion algebras, available at http://www.i2m.univ-amu.fr/perso/ david.kohel/alg/doc/AlgQuat.pdf.

[15] T. Y. Lam, Introduction to quadratic forms over fields, American Mathematical Society, Providence, 2005. 
[16] S. Lang, Algebra, Springer, New York, 2002.

[17] A. Ledet, Brauer type embedding problems, American Mathematical Society, Providence, 2005.

[18] B. Linowitz, Selectivity in quaternion algebras, J. Number Theory 132 (2012), 14251437.

[19] D. Marcus, Number Fields, Springer, New York-Heidelberg, 1977.

[20] L. Rowen and D. J. Saltman, Tensor products of division algebras and fields, J. Algebra 394 (2013), 296-309.

[21] M. Pohst and H. Zassenhaus, Algorithmic algebraic number theory, Cambridge University Press, 1989.

[22] D. Savin, About division quaternion algebras and division symbol algebras, Carpathian J. Math. 32 (2016), 233-240.

[23] D. Savin, About split quaternion algebras over quadratic fields and symbol algebras of degree $n$, Bull. Math. Soc. Sci. Math. Roumanie 60 (2017), 307-312.

[24] J. P. Serre, Local fields, Springer, New York, 1979.

[25] S. V. Vostokov, Explicit formulas for the Hilbert symbol, in: Geometry and Topology Monographs, Vol. 3: Invitation to higher local fields, eds. I. Fesenko and M. Kurihara, Geom. Topol. Publ., Coventry, 2000, 81-89.

[26] M. Waldschmidt, P. Moussa, J. M. Luck and C. Itzykson (eds.), From number theory to physics, Springer, Berlin, 1992.

V. Acciaro

Dipartimento di Economia

Università di Chieti-Pescara

Viale della Pineta, 4, 65127 Pescara

Italy

E-mail: v.acciaro@unich.it

D. Savin

Faculty of Mathematics and Computer Science

Transilvania University of Brașov

Iuliu Maniu street 50, Brașov 500091

Romania

E-mail: diana.savin@unitbv.ro \& dianet72@yahoo.com

M. Taous

Department of Mathematics, Faculty of Sciences and Technology

Moulay Ismail University

Errachidia

Morocco

E-mail: taousm@hotmail.com

A. Zekhnini

Department Mathematics and Informatics, Sciences Faculty, Oujda

Mohammed First University

Nador

Morocco

E-mail: zekha1@yahoo.fr

Received: 5.3.2020.

Revised: 14.11.2020. 